\title{
Radio Wavelength Constraints on the Sources of the Far Infrared Background
}

\author{
D. B. Haarsma ${ }^{1}$ and R. B. Partridge ${ }^{2}$ \\ Haverford College, Haverford, PA 19041
}

\begin{abstract}
The cosmic far infrared background detected recently by the COBE-DIRBE team is presumably due, in large part, to the far infrared (FIR) emission from all galaxies. We take the well-established correlation between FIR and radio luminosity for individual galaxies and apply it to the FIR background. We find that these sources make up about half of the extragalactic radio background, the other half being due to AGN. This is in agreement with other radio observations, which leads us to conclude that the FIR-radio correlation holds well for the very faint sources making up the FIR background, and that the FIR background is indeed due to star-formation activity (not AGN or other possible sources). If these star-forming galaxies have a radio spectral index between 0.4 and 0.8 , and make up 40 to $60 \%$ of the extragalactic radio background, we find that they have redshifts between roughly 1 and 2, in agreement with recent estimates by Madau et al. of the redshift of peak star-formation activity. We compare the observed extragalactic radio background to the integral over the $\log N-\log S$ curve for star-forming radio sources, and find that the slope of the curve must change significantly below about $1 \mu \mathrm{Jy}$. At $1 \mu \mathrm{Jy}$, the faint radio source counts predict about 25 sources per square arcminute, and these will cause SIRTF to be confusion limited at $160 \mu \mathrm{m}$.
\end{abstract}

Subject headings: cosmology: observations - diffuse radiation — galaxies: evolution — infrared: galaxies — stars: formation — radio continuum:galaxies

\footnotetext{
${ }^{1}$ dhaarsma@haverford.edu

${ }^{2}$ bpartrid@haverford.edu
} 


\section{Introduction}

The COBE-DIRBE team has recently announced the detection of the cosmic far infrared background (Hauser et al. 1998). The team interprets this flux as the sum of far infrared (FIR) emission from all galaxies, presumably mostly reemission from warm dust (although other interpretations have been made, e.g. Bond, Carr, \& Hogan 1986, 1991). The DIRBE results thus provide important constraints on the star formation history. In this letter, we use the DIRBE flux measurement, in conjunction with radio wavelength observations, to explain some of the radio extragalactic surface brightness, to estimate the redshift of the sources producing the DIRBE background, and to constrain radio source counts.

At $240 \mu \mathrm{m}$ and $140 \mu \mathrm{m}$, the fluxes measured by the DIRBE team are $14 \pm 3 \times 10^{-9} \mathrm{Wm}^{-2}$ ster $^{-1}$ and $25 \pm 7 \times 10^{-9} \mathrm{Wm}^{-2}$ ster $^{-1}$, respectively (Hauser et al. 1998). These values are in agreement with the FIRAS spectrum over 125-2000 $\mu \mathrm{m}$ reported by Fixsen et al. (1998). We have elected to use these more recent results rather than the tentative detection reported by Puget et al. (1996). The Hauser et al. measurement, and the upper limits at $60 \mu \mathrm{m}$ and $100 \mu \mathrm{m}$, show that the flux density has a nearly flat spectrum,

and thus we take the flux density to be $S_{\mathrm{FIR}}=1.15 \pm 0.20 \times 10^{-20} \mathrm{Wm}^{-2} \mathrm{ster}^{-1} \mathrm{~Hz}^{-1}$ between $140 \mu \mathrm{m}$ and $240 \mu \mathrm{m}$ in our subsequent calculations.

\section{Prediction of the Extragalactic Radio Background}

We begin with the assumption that the well-established correlation between the far infrared and $20 \mathrm{~cm}$ radio flux densities of galaxies,

$$
\frac{S_{80 \mu \mathrm{m}}}{S_{20 \mathrm{~cm}}}=10^{2.34}
$$

(Helou, Soifer, \& Rowan-Robinson 1985; Condon, Andreson, \& Helou 1991) holds for whatever sources produce the bulk of DIRBE background. The relationship has been observed for galaxies with a range of $10^{4}$ in FIR flux density, from normal spirals to ultraluminous IRAS galaxies (Cox et al. 1988; Crawford et al. 1996). The correlation does not, however, hold for galaxies in which the radio emission is not associated with star formation, such as AGN or classic radio galaxies. The physical origin of the correlation, while not completely understood, is probably due to star-formation processes, namely dust reemission in the far infrared, and supernova remnant synchrotron emission or thermal emission in the radio (see Condon 1992 for a review). Thus, it is reasonable to suppose that the relationship holds for the higher redshift, faint galaxies producing the DIRBE 
background.

A substantial fraction of the DIRBE background, and the associated radio emission, may be redshifted. Restframe emission at $80 \mu \mathrm{m}$ from sources in the range $0.75<z<2.0$ would be detected by DIRBE between $140 \mu \mathrm{m}$ and $240 \mu \mathrm{m}$. Since the luminosity density and star formation rate peak approximately in this same interval (Madau et al. 1996), we will initially take $z=1.0$ as a typical redshift for the sources producing the DIRBE background. Thus the radio flux emitted by these sources at $20 \mathrm{~cm}(1.4 \mathrm{GHz})$ will be observed at $40 \mathrm{~cm}$ (750 MHz).

Using the FIR-radio correlation, we calculate that the surface brightness at $40 \mathrm{~cm}$ due to star-forming galaxies is $S_{40 \mathrm{~cm}}^{*}=10^{-2.34} S_{\mathrm{FIR}} \simeq 5300 \mathrm{Jy} / \mathrm{ster}$, or about $0.5 \mathrm{mJy} / \mathrm{amin}^{2}$. The asterisk is used to indicate emission from star-forming galaxies (i.e., emission from AGN and the CMB is not included). The corresponding brightness temperature is $T_{40 \mathrm{~cm}}^{*}=0.31 \mathrm{~K}$.

To compare this to the observed brightness temperature, we scale this result to other radio bands using an appropriate spectral index $\alpha$ (where $S_{\nu} \propto \nu^{-\alpha}$ ) for the source population. Windhorst et al. (1993) compiled various surveys of faint radio sources at $50 \mathrm{~cm}(600 \mathrm{MHz})$ and $75 \mathrm{~cm}(400 \mathrm{MHz})$, and found the median spectral index between these bands and $6 \mathrm{~cm}(5 \mathrm{GHz})$ to be $\alpha \sim 0.7$ for faint sources, a value we will assume unless noted. Thus, the brightness temperature at $170 \mathrm{~cm}(178 \mathrm{MHz})$ due to the DIRBE sources is $T_{170 \mathrm{~cm}}^{*} \sim 15 \mathrm{~K}$.

We may compare this prediction with the observations of Bridle (1967), who found $T_{170 \mathrm{~cm}}=30 \pm 7 \mathrm{~K}$ for the entire extragalactic brightness temperature, including emission from AGN and $2.728 \mathrm{~K}$ from the cosmic microwave background (Fixsen et al. 1996). Subtracting the temperature of the CMB, we see that source of the DIRBE background explains more than half of the observed radio sky brightness due to all extragalactic sources. If we adopt a flatter spectral index for the radio sources of $\alpha=0.4$, the fraction of the observed radio background explained by the DIRBE sources drops to about a third. Thus the simple assumption that the FIR-radio correlation holds for the DIRBE sources allows us to explain a significant fraction of the observed radio brightness of the sky.

Our results are consistent with observations at other radio bands, which show that about half of the extragalactic radio background is due to star-forming galaxies. For instance, Condon (1989) estimates that at $20 \mathrm{~cm}$, AGN and star-forming galaxies contribute almost equally to the total spectral power density.

An analysis of radio source counts allows us to reinforce this conclusion. The counts are the sum of the two populations (star-forming galaxies and AGN), with the bulk of the 
AGN brighter than the bulk of the star-forming galaxies (see, e.g., Windhorst et al. 1993). We can thus take all the bright sources as a proxy for AGN, and then estimate the total fraction of the surface brightness of the radio sky that AGN would contribute. At $20 \mathrm{~cm}$, the dividing line between the two populations has been estimated as 1 mJy (Condon 1989) and $9 \mathrm{mJy}$ (Kron, Koo, \& Windhorst 1985). Ryle (1968) uses a lower limit of $10 \mathrm{mJy}$ at $75 \mathrm{~cm}$ (which corresponds to $4 \mathrm{mJy}$ at $20 \mathrm{~cm}$ for $\alpha=0.7$ ), and integrates over $d N / d S$ to find $T_{75 \mathrm{~cm}}^{\mathrm{AGN}} \sim 1.4 \mathrm{~K}$ for the contribution to the background from AGN. We can compare this to the total radio background by scaling Bridle's 1967 measurement at $170 \mathrm{~cm}$ and correcting for the $\mathrm{CMB}$ contribution to find $T_{75 \mathrm{~cm}}=2.9 \pm 0.7 \mathrm{~K}$ for the total radio brightness. Thus, the AGN explain about half of the total radio background, leaving the other half due to star-forming systems.

Since observations at other radio bands have also found that about half of the extragalactic radio background is produced by star-forming galaxies, we conclude that the assumed FIR-radio correlation holds for the sources responsible for the DIRBE background. Since the tight FIR-radio correlation seen in individually observed galaxies at lower redshift is ascribed to star formation, our results bolster the argument that star formation is the cause of most of the FIR background at the presumably higher redshifts of the DIRBE sources.

\section{The Redshift of Sources Producing the DIRBE Background}

We have so far assumed that both the FIR and the radio flux are produced at $z=1$. If the bulk of the emission originates at a different redshift, we will need to make the appropriate K-corrections. It is convenient that the DIRBE measurements and limits have found the FIR background to have a flat spectrum between 140 and $240 \mu \mathrm{m}$ (see $\S 1$ ), so that to first order we can ignore the FIR K-corrections in the range $0.75<z<2.0$ for $80 \mu \mathrm{m}$ emission. At radio wavelengths, the spectral index must be used to make the K-corrections.

To find the redshift of the DIRBE sources, we once again assume the FIR-radio correlation. We then scale the radio emission to an observing wavelength of $170 \mathrm{~cm}$, and write $A$ as the fraction $S_{170 \mathrm{~cm}}^{*} / S_{170 \mathrm{~cm}}$ of the observed flux density which we ascribe to star-forming galaxies, giving

$$
S_{170 \mathrm{~cm}}=\frac{1}{A} S_{\mathrm{FIR}} 10^{-2.34}\left(\frac{170 \mathrm{~cm}}{20 \mathrm{~cm}(1+z)}\right)^{\alpha} .
$$

Solving this expression for the redshift, and substituting in the measured values of $T_{170 \mathrm{~cm}}$ 
(see $\S 2$ ) and $S_{\text {FIR }}$ (see $\S 1$, we find the dimensionless relationship

$$
A\left(\frac{1+z}{8.5}\right)^{\alpha}=0.20 \pm 0.06
$$

which is plotted in Figure 1.

For our nominal values of $A=0.5$ and $\alpha=0.7$, we find $z+1 \sim 2.3 \pm 1.0$, in agreement with our initial assumption of $z=1$. While the uncertainty is large, this value is in agreement with the redshift of peak star formation of $z \sim 1.5$ found by Madau et al. (1996). The measured redshifts of individual faint radio galaxies (less than $1 \mathrm{mJy}$ ) are typically $z \sim 0.5-0.75$ Condon 1989; Windhorst et al. 1993; Richards et al. 1998); our value of $z$ is somewhat higher than this estimate, but this is reasonable since the fainter galaxies making up the DIRBE background should be at higher redshifts than brighter galaxies detected individually.

Let us now assume reasonable values of $z$ and $\alpha$ and consider the allowable range of the parameter $A$. We can assume that the bulk of star formation is certainly more recent than the epoch corresponding to $z=8$. Then Figure 1 shows that the fraction of the radio background from star forming galaxies, $A$, must be greater than $20 \%$ for any reasonable spectral index. Since $z$ must be greater than zero, we can also find upper limits on $A$ : for $\alpha=0.7, A$ must be less than about $90 \%$, and for $\alpha=0.4$, less than $50 \%$. If star formation peaks in the interval $1<z<2$ and $\alpha \sim 0.7, A$ is tightly constrained to the interval $40-60 \%$.

\section{Constraints on Faint Radio Source Counts}

The brightness temperature of the radio background, corrected for the CMB, is due to combined flux of the faint radio galaxies and thus is related to the number counts of these galaxies,

$$
T=\frac{\lambda^{2}}{2 k} \int_{S_{\min }}^{S_{\max }} S \frac{d N}{d S} d S .
$$

We can take the number counts to be of the form $d N / d S=C S^{\gamma}$, where $\gamma$ is generally less than -2 for this population at radio wavelengths (see eq. [5]). For $\gamma<-2$, faint sources dominate the contribution to the background temperature. It has also long been recognized that, for $\gamma<-2$, the total source counts and the radio sky brightness will (slowly) diverge as $S \rightarrow 0$ (see, e.g., Windhorst et al. 1993). Therefore, there must be a cutoff or change in

slope of the $\log N-\log S$ curve at some minimum value, $S_{\min }$. To estimate $S_{\min }$ we can use the radio brightness temperature due to the DIRBE star-forming galaxies in conjunction with the $\log N-\log S$ relation for faint radio sources. 
Very deep VLA observations at $3.6 \mathrm{~cm}$ (8 GHz) (Windhorst et al. 1993; Windhorst et al. 1995; Fomalont et al. 1997; Kellermann et al. 1998) have been used to determine the number count power law,

$$
\frac{d N}{d S}=-4.6 \pm 0.7\left(\frac{S}{1 \mathrm{Jy}}\right)^{-2.3 \pm 0.2} \mathrm{Jy}^{-1} \mathrm{ster}^{-1},
$$

which is valid for flux densities in the range $14.5 \mu \mathrm{Jy}<S<1000 \mu \mathrm{Jy}$. At these faint flux levels, there is little contamination from AGN, i.e. the sources are nearly all star forming galaxies (Condon 1989). For $\gamma<-2$, the definite integral is dominated by $S_{\min }$ and is independent of $S_{\max }$ for large values of $S_{\max }$; we take $S_{\max }$ to be infinity. Thus, the integral over these number counts will yield the radio brightness temperature due to the DIRBE sources found above. Scaling this temperature to $3.6 \mathrm{~cm}$, integrating, and solving for $S_{\min }$, we find

$$
S_{\text {min }}=\left[\frac{2+\gamma}{C}\left(\frac{S_{\mathrm{FIR}}}{\mathrm{Jy}}\right) 10^{-2.34}\left(\frac{3.6 \mathrm{~cm}}{20 \mathrm{~cm}(1+z)}\right)^{\alpha}\right]^{\frac{1}{2+\gamma}} .
$$

For $\alpha=0.7, z=1.0$, the observed value of $S_{\mathrm{FIR}}$, and $\gamma$ and $C$ from equation (5), we find $S_{\text {min }} \gtrsim 1 \mu \mathrm{Jy}$ at $3.6 \mathrm{~cm}$.

It is useful to compare our estimate of $S_{\min }$, based on the far infrared background, with other estimates at $3.6 \mathrm{~cm}$, such as those made by Windhorst et al. (1993). In order for the radio background due to galaxies to not distort the spectrum of the cosmic microwave background (CMB), they found $S_{\min } \geq 20 \mathrm{nJy}$ at $3.6 \mathrm{~cm}$. They also found that if $S_{\min }$ fell below $300 \mathrm{nJy}$, the optical counterparts of these faint radio sources would exceed the V band counts of field galaxies. The limit of $S_{\min } \geq 1 \mu \mathrm{Jy}$ we have found is consistent with these, but more restrictive, and has more interesting observational consequences.

An RMS sensitivity of $1.5 \mu \mathrm{Jy}$ has been reached in recent VLA observations at $3.6 \mathrm{~cm}$ (Partridge et al. 1997), and our value of $S_{\min }$ indicates that there are few radio sources below this flux density, that is, the slope of the $\log N-\log S$ curve changes significantly at $S_{\text {min }}$. The present VLA observations have thus detected the bulk of all radio sources in the Universe.

The value of $S_{\min }$ (at $z \sim 1$ ) can also be compared to the knee in the luminosity function for local faint radio sources. At $20 \mathrm{~cm}$, the slope turns over around $10^{22.4} \mathrm{~W} / \mathrm{Hz}$ (Condon 1989). A source at $z=1$ with a flux density of $1 \mu \mathrm{Jy}$ at $3.6 \mathrm{~cm}$ would have a $20 \mathrm{~cm}$ luminosity of about $10^{21.7} h^{-2} \mathrm{~W} / \mathrm{Hz}$ (assuming $\alpha=0.7$ and an Einstein-deSitter universe). Thus, the turnover point at $z=1$ is very similar to the local value, especially for a Hubble parameter of $h \sim 0.6$.

We can also consider the fluctuations in the radio background due to these sources. The 
number counts (eq. [5]) at the $1 \mu \mathrm{Jy}$ level indicate that there should be about 25 sources per $\operatorname{armin}^{2}$, or about $3 \times 10^{8}$ sources/steradian. The average separation of $1 \mu \mathrm{Jy}$ sources is thus about $14^{\prime \prime}$, and we would expect substantial fluctuation in the surface brightness of the radio sky on this angular scale. The RMS due to the sources can in principle be calculated from the number counts, assuming the sources are distributed randomly on the sky. When the number counts follow a power law distribution, as in equation (5), the RMS is determined by the upper and lower limits on the source flux densities, $S_{\min }$ and $S_{\max }$. For a power law with $\gamma>-3$, the RMS is unfortunately dominated by the value of $S_{\max }$, which is not well known for this population (the bright star-forming galaxies are comparable to the faint AGN, making it difficult to determine the precise upper bound on the population). Thus, it goes beyond the scope of this paper to estimate the RMS in the radio background due to this population. We concur, however, with the conclusions of Partridge et al. (1997) and Mitchell \& Condon (1985), that these faint radio sources do contribute substantially to the RMS fluctuations in the microwave sky. Others have noted that at the higher frequencies and lower angular resolutions planned for satellite- and ground-based searches for CMB fluctuations, the faint sources that produce the FIR background should present no problems (Windhorst et al. 1993; Toffolatti et al. 1995), unless the sources are strongly clustered.

We can also ask how these sources will appear in number counts and high resolution maps in the far infrared. For $\alpha$ between 0.4 and 0.7 , and $z=1$, we use the FIR-radio correlation to calculate that $3.6 \mathrm{~cm}$ sources with flux density $S_{\min }(1 \mu \mathrm{Jy})$ will appear as $600-1200 \mu \mathrm{Jy}$ sources at $160 \mu \mathrm{m}$. The number density of these faint FIR sources can be compared to the semi-analytic models of galaxy formation of Guiderdoni et al. (1998), who predict the FIR background and the faint galaxy counts for various FIR wavelengths. One of their models finds a number density of $10^{8}$ sources/steradian for $1 \mathrm{mJy}$ sources at $175 \mu \mathrm{m}$, which is close to our estimate of $3 \times 10^{8}$ sources/steradian. At $160 \mu \mathrm{m}$, the longest SIRTF-MIPS wavelength, SIRTF will have $15^{\prime \prime}$ pixels and an angular resolution of about $30^{\prime \prime}$ (Rieke, Young, \& Gautier 1996), larger than the average separation of $14^{\prime \prime}$ between the faintest sources. Thus, SIRTF will not be able to resolve the individual sources responsible for the DIRBE flux, and will be confusion limited. This is in reasonable agreement with the Monte Carlo simulations of Rieke et al. (1996), who find a confusion limit for SIRTF of $1500 \mu \mathrm{Jy}$.

\section{Conclusions}

Using the well-established correlation between the FIR and radio luminosity of individual galaxies, we have extrapolated the FIR background detected by DIRBE to radio 
wavelengths. This has allowed us to derive several properties of the sources making up the FIR background:

1. The radio emission from the sources makes up about half of the observed extragalactic radio background (about $0.3 \mathrm{~K}$ at wavelengths around $40 \mathrm{~cm}$, excluding the cosmic microwave background).

2. Since this finding is in agreement with other radio observations, the FIR-radio correlation holds even for the very faint sources making up the DIRBE background. This implies that the FIR background between about 140 and $240 \mu \mathrm{m}$ is dominated by star-formation, not AGN activity.

3. The typical redshift and spectral index $\alpha$ of these sources, and the contribution they make to the radio background are related by equation (3). For reasonable values of the spectral index (0.4 to 0.7$)$ and the fraction of the radio background (40-60\%), we find the redshift of these sources to be roughly between 1 and 2, in agreement with the Madau et al. (1996) estimate of the redshift of peak star formation.

4. By extrapolating the radio $\log N-\log S$ curve at $3.6 \mathrm{~cm}$ to fainter flux densities, we estimate that most of the DIRBE flux is produced by sources whose $3.6 \mathrm{~cm}$ flux density is $z 1 \mu \mathrm{Jy}$, and that the number density of $1 \mu \mathrm{Jy}$ sources is about $25 / \mathrm{arcmin}^{2}$. These sources will cause SIRTF to be confusion limited around $160 \mu \mathrm{m}$.

We thank Steve Boughn, Jim Condon, Alberto Franceschini, Michael Hauser, Ken Kellermann, Rogier Windhorst, and our referee for their helpful comments. This work was supported by NSF grant AST96-16971. 


\section{REFERENCES}

Bond, J. R., Carr, B. J., \& Hogan, C. J. 1986, ApJ 306, 428

Bond, J. R., Carr, B. J., \& Hogan, C. J. 1991, ApJ 367, 420

Bridle, A. H. 1967, MNRAS 136, 219

Condon, J. J. 1989, ApJ 338, 13

Condon, J. J. 1992, ARA\&A 30, 575

Condon, J. J., Anderson, M. L., \& Helou, G. 1991, ApJ 376, 95

Cox, M. J., Eales, S. A. E., Alexander, P., \& Fitt, A. J. 1988, MNRAS 235, 1227

Crawford, T., Marr, J., Partridge, B., \& Strauss, M. A. 1996, ApJ 460, 225

Fixsen, D. J., Cheng, E. S., Gales, J. M., Mather, J. C., Shafer, R. A., \& Wright, E. L. 1996, ApJ 473, 576

Fixsen, D. J., Dwek, E., Mather, J. C., Bennett, C. L., \& Shafer, R. A. 1998, ApJ in press

Fomalont, E. B., Kellermann, K. I., Richards, E. A., Windhorst, R. A., \& Partridge, R. B. 1997, ApJ 475, L5

Guiderdoni, B., Hivon, E., Bouchet, F. R., \& Maffei, B. 1998, MNRAS 295, 877

Hauser, M. G. et al. 1998, submitted

Helou, G., Soifer, B. T., \& Rowan-Robinson, M. 1985, ApJ 298, L7

Kellermann, K. I. et al. 1998, in preparation

Kron, R. G., Koo, D. C., \& Windhorst, R. A. 1985, A\&A 146, 38

Madau, P., Ferguson, H. C., Dickinson, M. E., Giavalisco, M., Steidel, C. C., \& Fruchter, A. 1996, MNRAS 283, 1388

Mitchell, K. J. \& Condon, J. J. 1985, AJ 90, 1957

Partridge, R. B., Richards, E. A., Fomalont, E. B., I.Kellermann, K., \& Windhorst, R. A. 1997, ApJ 483, 38

Puget, J.-L., Abergel, A., Bernard, J.-P., Boulanger, F., Burton, W. B., Désert, F.-X., \& Hartmann, D. 1996, A\&A 308, L5 
Richards, E. A., Kellermann, K. I., Fomalont, E. B., Windhorst, R. A., \& Partridge, R. B. 1998, AJ, in press

Rieke, G. H., Young, E. T., \& Gautier, T. N. 1996, Space Science Reviews 74, 17

Ryle, M. 1968, ARAESA 6, 249

Toffolatti, L. et al. 1995, Astrophysics Letters \&3 Commentary 32, 125

Windhorst, R. A., Fomalont, E. B., Kellermann, K. I., Partridge, R. B., Richards, E., Franklin, B. E., Pascarelle, S. M., \& Griffiths, R. E. 1995, Nature 375, 471

Windhorst, R. A., Fomalont, E. B., Partridge, R. B., \& Lowenthal, J. D. 1993, ApJ 405, 498 


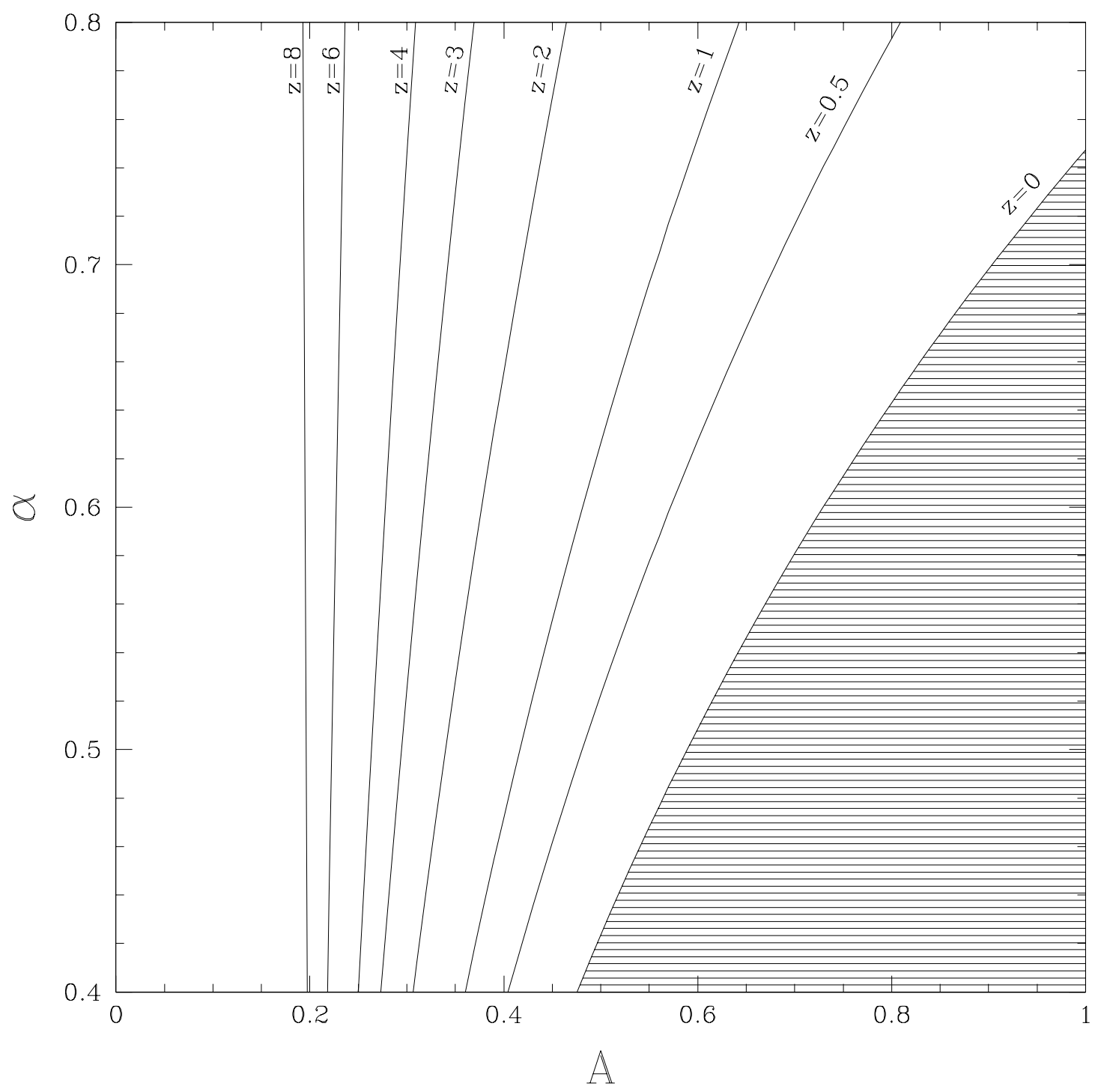

Fig. 1.- Relationship between the radio spectral index $\alpha$, the ratio of star-formation flux to the total radio background $A$, and the typical redshift $z$ for the sources making up the FIR background. See equation (3). 